\title{
Added value of dedicated axillary hybrid 18F-FDG PET/MRI for improved axillary nodal staging in clinically node-positive breast cancer patients: a feasibility study
}

\author{
Thiemo J. A. van Nijnatten ${ }^{1,2,3}$ (D) B. Goorts ${ }^{1,2,3}$ • S. Vöö ${ }^{1}$ - M. de Boer $^{3,4}$. \\ L. F. S. Kooreman ${ }^{3,5}$ - E. M. Heuts ${ }^{2}$ • J. E. Wildberger ${ }^{1}$ - F. M. Mottaghy ${ }^{1,6}$. \\ M. B. I. Lobbes ${ }^{1,3}$ - M. L. Smidt ${ }^{2,3}$
}

Received: 30 May 2017 / Accepted: 31 August 2017 / Published online: 14 September 2017

(C) The Author(s) 2017. This article is an open access publication

\begin{abstract}
Purpose To investigate the feasibility and potential added value of dedicated axillary 18F-FDG hybrid PET/MRI, compared to standard imaging modalities (i.e. ultrasound [US], MRI and PET/CT), for axillary nodal staging in clinically node-positive breast cancer.

Methods Twelve patients with clinically node-positive breast cancer underwent axillary US and dedicated axillary hybrid 18F-FDG PET/MRI. Nine of the 12 patients also underwent whole-body PET/CT. Maximum standardized uptake values (SUVmax) were measured for the primary breast tumor and the most FDG-avid axillary lymph node. A positive axillary lymph node on dedicated axillary hybrid PET/MRI was defined as a moderate to very intense FDG-avid lymph node. The diagnostic performance of dedicated axillary hybrid PET/ MRI was calculated by comparing quantitative and its qualitative measurements to results of axillary US, MRI and PET/
\end{abstract}

Thiemo J. A. van Nijnatten

Thiemovn@gmail.com

1 Department of Radiology and Nuclear Medicine, Maastricht University Medical Center+, P.O. Box 5800, 6202

AZ Maastricht, The Netherlands

2 Department of Surgery, Maastricht University Medical Center+, Maastricht, The Netherlands

3 GROW - School for Oncology and Developmental Biology, Maastricht University Medical Center+, Maastricht, The Netherlands

4 Division of Internal Medicine, Department of Medical Oncology, Maastricht University Medical Center+, Maastricht, The Netherlands

5 Department of Pathology, Maastricht University Medical Center+, Maastricht, The Netherlands

6 Department of Nuclear Medicine, University Hospital, RWTH Aachen University, Aachen, Germany
CT. The number of suspicious axillary lymph nodes was subdivided as follows: N0 (0 nodes), N1 (1-3 nodes), N2 (4-9 nodes) and N3 ( $\geq 10$ nodes).

Results According to dedicated axillary hybrid PET/MRI findings, seven patients were diagnosed with N1, four with $\mathrm{N} 2$ and one with N3. With regard to mean SUVmax, there was no significant difference in the primary tumor $(9.0[ \pm 5.0]$ vs. $8.6[ \pm 5.7], p=0.678)$ or the most FDG-avid axillary lymph node $(7.8[ \pm 5.3]$ vs. $7.7[ \pm 4.3], p=0.767)$ between dedicated axillary PET/MRI and PET/CT. Compared to standard imaging modalities, dedicated axillary hybrid PET/MRI resulted in changes in nodal status as follows: $40 \%$ compared to US, $75 \%$ compared to T2-weighted MRI, $40 \%$ compared to contrastenhanced MRI, and 22\% compared to PET/CT.

Conclusions Adding dedicated axillary 18F-FDG hybrid PET/MRI to diagnostic work-up may improve the diagnostic performance of axillary nodal staging in clinically nodepositive breast cancer patients.

Keywords Breast cancer · Lymph node imaging · Hybrid PET/MRI · Axilla

\section{Introduction}

Breast cancer is one of the most prevalent types of invasive cancer among women [1]. The involvement of axillary lymph nodes is an important prognostic factor, as 5-year overall survival is reduced from $98 \%$ to $85 \%$ in the case of lymph node metastases [2]. Therefore, European guidelines recommend preoperative axillary imaging to differentiate between patients with and without clinically node-positive breast cancer [3]. Accurate lymph node staging is important, as it affects the 
type of axillary surgery and indications for systemic therapy and radiotherapy fields [4].

In addition to differentiating between node-negative and node-positive disease, an equally important consideration is the number of suspicious nodes. Current treatment plans are based on the number of suspicious lymph nodes (i.e. one to three versus four or more) according to imaging findings and histopathological confirmation prior to the start of therapy [3]. Preoperative axillary imaging currently involves the use of axillary ultrasound (US), with tissue sampling in the case of suspicious nodal findings. Schipper et al. demonstrated that more than $50 \%$ of patients with one to three suspicious lymph nodes on US had four or more lymph node metastases at surgery [5]. This percentage decreased to $11-15 \%$ when standard breast MRI was used for axillary nodal staging [6]. In addition, the use of a dedicated axillary MR protocol in place of a standard breast MR protocol further improves the diagnostic performance of MRI for nodal staging [7]. Research suggests that PET/CT has the highest accuracy for preoperative nodal staging: Koolen et al. demonstrated that $98 \%$ of all patients with suspicious axillary lymph nodes on PET/CT would eventually have axillary lymph node metastases after surgery [8].

Hybrid PET/MRI allows for the simultaneous use of the promising axillary nodal staging techniques PET and MRI [9]. Therefore, the aim of this study was to investigate the feasibility and potential added value of dedicated axillary $18 \mathrm{~F}$ FDG hybrid PET/MRI compared to standard imaging modalities (i.e. axillary US, MRI and PET/CT) for axillary nodal staging in patients with clinically node-positive breast cancer.

\section{Material and methods}

\section{Setting and patients}

After approval of the study by the local medical ethical committee, a total of 12 patients provided signed informed consent to undergo dedicated axillary hybrid PET/MRI. Inclusion criteria were patients with histopathologically confirmed clinically node-positive breast cancer. Exclusion criteria were contraindications for either MRI, including the contrast agent gadobutrol, or PET, including radiotracer $18 \mathrm{~F}$-fluorodeoxyglucose (18F-FDG). Prior to inclusion, patients underwent axillary US. In addition, all patients were scheduled for MR imaging and, depending on the number of suspicious lymph nodes (i.e. 1-3 vs. 4+), for PET/CT as well [10].

\section{Axillary ultrasound}

Axillary US exams were performed by the radiologist on call, using an ultrasound system with a linear 2- to $17-\mathrm{MHz}$ array transducer (iU22-xMATRIX, Philips Healthcare, Best, the Netherlands). Criteria for suspicious axillary lymph nodes included diffuse cortical thickening, focal cortical mass and/ or thickening, and loss of fatty hilum [11]. During axillary US, tissue sampling of suspicious axillary lymph nodes was performed. In the case of multiple suspicious nodes, only the most suspicious node was sampled, and the number of suspicious axillary lymph nodes was reported.

\section{PET/CT}

In the Netherlands, PET/CT exams can be considered in the case of stage III/IV breast cancer [10, 12]. After patients had fasted for a period of at least $4 \mathrm{~h}$ and had a blood glucose level $<10 \mathrm{mmol} / \mathrm{L}$, an intravenous injection of $18 \mathrm{~F}-\mathrm{FDG}$ was administered at a dose of $2 \mathrm{MBq} / \mathrm{kg}$ body weight, followed by a resting period of $60 \pm 10 \mathrm{~min}$. Patients were placed in supine position with elevated arms in the PET/CT scanner (Gemini TF, Philips Healthcare, Best, the Netherlands). A low-dose CT scan $(120 \mathrm{kV}, 30 \mathrm{mAs}$, slice thickness $4 \mathrm{~mm})$ from head to thigh was performed, followed by the PET acquisition ( 3 min per bed position). CT images were reconstructed using filtered back-projection. PET images were reconstructed using the BLOB-OS-TF time-of-flight algorithm provided by the manufacturer, with a voxel size of $4 \times 4 \times 4 \mathrm{~mm}^{3}$.

\section{Hybrid PET/MRI}

Directly following whole-body PET/CT, state-of-the-art dedicated axillary hybrid PET/MRI images were acquired on a $3.0 \mathrm{~T}$ Biograph mMR integrated PET/MRI system (Siemens Healthineers, Erlangen, Germany). Dedicated axillary MRI was performed using a six-channel anterior body coil (Siemens Healthineers), which was placed on the ipsilateral shoulder. Patients were placed in prone position with both arms elevated.

In three cases, no whole-body PET/CT was performed. These patients underwent dedicated axillary hybrid PET/ MRI after administration of 18F-FDG at a dose of $2 \mathrm{MBq} /$ $\mathrm{kg}$ body weight and a resting period of $60 \pm 10 \mathrm{~min}$, with identical fasting time and a blood glucose level $<10 \mathrm{mmol} /$ $\mathrm{L}$ as with $\mathrm{PET} / \mathrm{CT}$.

The dedicated axillary hybrid PET/MRI protocol consisted of an unenhanced T2-weighted (T2W) sequence, contrastenhanced T1-weighted sequence (TW1), and a fusion sequence of PET images with the unenhanced T2W sequence. All sequences were performed in coronal view.

More specifically, a 2D standard T2W sequence without fat suppression was performed first using the following scan parameters: repetition time (TR) $2000 \mathrm{~ms}$; echo time (TE) $118 \mathrm{~ms}$; variable degree flip angle; turbo spin echo (TSE) factor 41 ; averages 1.4 ; voxel size $1.1 \times 1.1 \times 1.1 \mathrm{~mm}$; field of view (FOV) $220 \times 220 \mathrm{~mm}^{2}$; matrix $192 \times 192$, resulting in an in-plane resolution of $1.15 \times 1.15 \mathrm{~mm}^{2}$. Next, gadobutrol (Gadovist ${ }^{\circledR}$, Bayer HealthCare, Berlin, Germany) contrast at a 
volume of $15 \mathrm{~cm}^{3}$ was administered intravenously. After 6 min, a 3D T1W sequence was acquired using the following parameters: TR $7.27 \mathrm{~ms}$; TE $2.46 \mathrm{~ms} ; 15^{\circ}$ flip angle; averages 2; voxel size $1.1 \times 1.1 \times 1.1 \mathrm{~mm}$; FOV $220 \times 220 \mathrm{~mm}^{2}$; matrix $192 \times 192$; resulting in an inplane resolution of $1.15 \times 1.15 \mathrm{~mm}^{2}$. Total acquisition time of the MRI exam was $20 \mathrm{~min}, 21 \mathrm{~s}$.

Three-dimensional iterative reconstruction was performed for all PET images of the hybrid PET/MRI system (number of iterations 3 ; subsets 21 ; voxel size $4.1 \times 2.6 \times 3.9 \mathrm{~mm}^{3}$; image matrix $172 \times 172$; zoom 1.0 ), with automatic attenuation correction through implementation of a four-compartment model attenuation map (Dixon-based $\mu$-map). Images were acquired in a single bed position within 15 min after the start of MRI acquisition on the hybrid PET/MRI system.

\section{Image evaluation}

All axillary lymph nodes on dedicated axillary MR, hybrid PET/MRI and PET/CT images were evaluated by a breast radiologist (M.B.I.L.) and nuclear medicine physician (S.V.). The breast radiologist has 7 years of dedicated experience in breast imaging, and the nuclear medicine physician has 9 years of experience in nuclear imaging.

First, the radiologist scored each axillary lymph node on dedicated axillary $\mathrm{T} 2 \mathrm{~W}$ images, according to the criteria previously defined by Baltzer er al., using a confidence scale from 0 (no lymph nodes) to 4 (definitely malignant) [13]. Lymph nodes with a score of 0-2 were considered benign, and those with a score of 3-4, malignant. Suspicious characteristics included irregular margins, inhomogeneous cortex, perifocal edema, absence of fatty hilum, asymmetry, and absence of chemical shift artifacts [13-15]. Next, contrastenhanced dedicated axillary T1W images were shown, which allowed the radiologist to adjust his score for each lymph node, if deemed necessary. Suspicious characteristics included absence of contrast hyperintensity of the lymph node and absence of an intact nodal border [15].

Second, the breast radiologist and nuclear medicine physician evaluated in consensus each axillary lymph node on dedicated axillary hybrid PET/MRI using a four-point system as previously described by Aukema et al. for the assessment of lymph nodes with $18 \mathrm{~F}-\mathrm{FDG}$ PET/CT: $0=$ similar to other surrounding lymph nodes, $1=$ slightly more intense than other lymph nodes, $2=$ moderately intense, $3=$ very intense [16]. Lymph nodes with a score of $0-1$ were considered benign, while a score of 2-3 was considered malignant. PET information was considered to be decisive regarding the number of suspicious axillary lymph nodes in the evaluation of dedicated axillary hybrid PET/MRI. The nuclear medicine physician further measured maximum standardized uptake values (SUVmax) of the primary breast tumor and the most FDGavid axillary lymph node on each dedicated axillary hybrid
PET/MRI exam. Finally, the nuclear medicine physician evaluated each axillary lymph node on 18F-FDG PET/CT, using the same four-point system [16], and measured SUVmax of the primary breast tumor and the most FDG-avid axillary lymph node on each PET/CT exam. Both PET/CT and PET/ MRI exams were shown anonymously and in randomized order to the nuclear physician for evaluation of the number of suspicious axillary lymph nodes.

\section{Pathology}

In this study, clinically node-positive disease was based on US-guided cytological and/or histological confirmation of axillary lymph node biopsy. In cases where US was performed in the referring hospital, the pathology of the axillary lymph node biopsy was revised and metastases were verified by the pathologist in our hospital (L.K.).

\section{Statistical analyses}

Statistical analyses were performed using SPSS software (version 22, IBM Corp., Armonk, NY, USA). Axillary nodal status was based on the number of suspicious axillary lymph nodes, as follows: none (N0), 1-3 (N1), 4-9 (N2), or 10 or more (N3), according to axillary US (including results of tissue sampling), dedicated axillary (PET)MRI and/or PET/CT, respectively. When the result of dedicated axillary hybrid PET/MRI led to either up- or down-staging of clinical nodal status compared to standard imaging modalities (US, MRI or $\mathrm{PET} / \mathrm{CT}$ ), it was considered a change in clinical nodal status according to dedicated axillary hybrid PET/MRI findings.

In patients who underwent both PET/CT and hybrid PET/ MRI, the Wilcoxon signed-rank test was used to compare the mean SUVmax of the primary tumor $( \pm$ standard deviation $[\mathrm{SD}])$ and the most FDG-avid axillary lymph node $( \pm \mathrm{SD})$ on PET/CT with hybrid PET/MRI. $P$-values (two-sided) $<0.05$ were considered statistically significant.

\section{Results}

\section{Patients}

A total of 12 consecutive patients with clinically node-positive breast cancer over a period from April 2016 to March 2017 were included in this feasibility study. The mean age was 49 years (range, $28-70$ years). In all patients, clinically positive nodal status was histopathologically confirmed with USguided biopsy. Eleven of 12 patients had invasive carcinoma of no special type; one patient had mixed lobular carcinoma with carcinoma of no special type. In addition, 9 of 12 patients underwent PET/CT. A more detailed overview of the general characteristics is shown in Table 1. 
Table 1 General patient characteristics

\begin{tabular}{ll} 
Mean age (years) (range) & $49(28-70)$ \\
Mean clinical tumor size (mm) (range) & $46(13-150)$ \\
cT-stage (\%) & \\
cT1 & $2(17 \%)$ \\
cT2 & $4(33 \%)$ \\
cT3 & $3(25 \%)$ \\
cT4 & $3(25 \%)$ \\
Multifocal tumor (\%) & \\
No & $8(67 \%)$ \\
Yes & $4(33 \%)$ \\
Tumor type (\%) & \\
Invasive carcinoma NST & $11(92 \%)$ \\
Mixed NST and lobular & $1(8 \%)$ \\
Tumor grade (\%) & \\
2 & $6(50 \%)$ \\
3 & $6(50 \%)$ \\
Hormone receptor/HER2 status & \\
ER/PR-positive, HER2-negative & $4(33 \%)$ \\
ER/PR-negative, HER2-positive & $1(8 \%)$ \\
Triple-negative & $2(17 \%)$ \\
ER/PR-positive, HER2-positive & $5(42 \%)$ \\
\hline
\end{tabular}

Abbreviations: cT-stage clinical tumor stage, NST no special type, ER estrogen, $P R$ progesterone, HER2 human epidermal growth factor receptor 2

\section{Nodal staging}

When compared to standard imaging modalities, dedicated axillary hybrid PET/MRI resulted in changes to clinical nodal status as follows: $40 \%$ according to US findings (4 of 10 patients), $75 \%$ according to T2W MRI findings (9 of 12 patients), $40 \%$ according to CE-T1W MRI findings (4 of 10 patients) and $22 \%$ according to PET/CT findings (2 of 9 patients), respectively. Differences between PET/CT and PET/ MRI findings were due to better delineation of the lymph nodes on the MRI component. A more detailed overview of all cases, including up- or down-staging of nodal status according to dedicated axillary hybrid PET/MRI findings, is provided in Table 2 .

In two patients, axillary US findings regarding the number of suspicious axillary lymph nodes was missing, because US was performed in the referring hospital. However, in addition, neither patient underwent contrast-enhanced T1W MRI, as this was refused by both patients.

Figures 1 and 2 illustrate examples of differences in the number of suspicious axillary lymph nodes between PET/ CT (Fig. 1) and T2W MRI (Fig. 2) when compared to dedicated axillary hybrid PET/MRI. The Appendix provides a complete overview of all axillary lymph nodes evaluated on dedicated axillary (PET/)MRI and PET/CT images.

\section{SUVmax measurements}

The mean SUVmax of the primary tumor and the most FDGavid axillary lymph node on PET/CT were $8.6( \pm 5.7)$ and 7.7 $( \pm 4.3)$, respectively. Comparable values were achieved on hybrid PET/MRI, with mean SUVmax of 9.0 ( \pm 5.0; $p=0.678)$ and $7.8( \pm 5.3 ; p=0.767)$ for the primary tumor and the most FDG-avid lymph node, respectively. Table 3 provides an overview of all SUVmax measurements on PET/CT and hybrid PET/MRI for each patient.

\section{Discussion}

The aim of this study was to investigate the feasibility and potential added value of dedicated axillary 18F-FDG hybrid PET/MRI compared to standard imaging modalities (i.e. US, MRI and PET/CT) for axillary nodal staging in patients with clinically node-positive breast cancer. The results of our study show that dedicated axillary $18 \mathrm{~F}-\mathrm{FDG}$ hybrid PET/MRI is clinically feasible, and resulted in a change in nodal status in $40-75 \%$ of patients when compared to US or MRI. When compared to PET/CT only, nodal status was changed in two of nine patients $(22 \%)$, although SUVmax measurements were comparable between the imaging modalities.

The importance of accurate differentiation in clinically node-positive patients - i.e. one to three versus four or more positive nodes - prior to start of treatment is a topic of debate, since adjuvant (radiation) therapy planning is based on pre-treatment axillary imaging findings in the case of neoadjuvant systemic therapy [17]. With regard to patients with limited axillary disease after surgery, the American College of Surgeons Oncology Group (ACOSOG) Z0011 trial demonstrated that there was no prognostic benefit in adjuvant axillary treatment in clinically node-negative patients treated with primary surgery [18]. On the other hand, patients with advanced axillary disease (i.e. 4+ positive nodes) do require adjuvant regional treatment $[17,19]$. Consequently, pre-treatment axillary imaging is becoming increasingly important as a means of truly identifying patients with four or more versus three or fewer positive nodes. However, long-term outcome for patients treated with additional therapy after the detection of a larger number of positive nodes - for instance, on PET/ $\mathrm{CT}$ as opposed to ultrasound-remains unclear.

In contrast to our study, Grueneisen et al. found no differences in PET/MRI findings compared to PET/CT and MRI for axillary nodal staging in breast cancer patients [20]. However, in that study, a (standard) breast PET/MRI protocol was used, unlike the state-of-the-art dedicated axillary PET/MRI protocol in our study. In addition, a previous study demonstrated that in approximately $40 \%$ of all patients who underwent standard breast 
Table 2 Clinical nodal status (including number of suspicious lymph nodes) according to US, PET/CT, T2W MRI, CE-T1W MRI and hybrid PET/MRI

\begin{tabular}{|c|c|c|c|c|c|}
\hline \multirow[t]{2}{*}{ Patient } & \multirow[t]{2}{*}{ US } & \multirow[t]{2}{*}{$\mathrm{PET} / \mathrm{CT}$} & \multicolumn{3}{|c|}{ Dedicated axillary (PET/)MRI } \\
\hline & & & T2W MRI & CE-T1W MRI & Hybrid PET/MRI \\
\hline 1 & N2 (4) & N2 (5) & N3 $(10) \downarrow$ & $\mathrm{N} 3(10) \downarrow$ & N2 (8) \\
\hline 2 & N1 (1) & - & $\mathrm{N} 0 \uparrow$ & N1 (1) & N1 (2) \\
\hline 3 & N1 (1) & - & $\mathrm{N} 2(5) \downarrow$ & $\mathrm{N} 2(7) \downarrow$ & N1 (1) \\
\hline 4 & N1 (3) $\uparrow$ & N2 (5) & $\mathrm{N} 3(10) \downarrow$ & N2 (9) & N2 (8) \\
\hline 5 & $\mathrm{~N}+(-)$ & N2 (9) & N1 (3) $\uparrow$ & - & $\mathrm{N} 2(7)$ \\
\hline 6 & N2 (6) $\downarrow$ & N1 (2) & $\mathrm{N} 3(25) \downarrow$ & $\mathrm{N} 3(11) \downarrow$ & N1 (2) \\
\hline 7 & $\mathrm{~N}+(-)$ & N2 (4) $\downarrow$ & N2 (4) $\downarrow$ & - & N1 (3) \\
\hline 8 & N1 (1) & N1 (3) & $\mathrm{N} 2(6) \downarrow$ & N2 (7) $\downarrow$ & N1 (3) \\
\hline 9 & $\mathrm{~N} 2(5) \uparrow$ & $\mathrm{N} 2(7) \uparrow$ & N3 (10) & N3 (13) & N3 (10) \\
\hline 10 & N1 (1) & N1 (1) & N1 (2) & N1 (2) & N1 (1) \\
\hline 11 & N1 (2) $\uparrow$ & - & N1 (3) $\uparrow$ & N2 (5) & N2 (4) \\
\hline 12 & N1 (1) & N1 (1) & N1 (2) & N1 (3) & N1 (1) \\
\hline
\end{tabular}

Abbreviations: PET positron emission tomography, CT computed tomography, $M R I$ magnetic resonance imaging, $T 2 W$ T2-weighted, $C E-T 1 W$ contrast-enhanced T1-weighted, $N 0$ no suspicious lymph nodes, $N 1$ 1-3 suspicious lymph nodes, N2 4-9 suspicious lymph nodes, N3 10 or more suspicious lymph nodes, $N+$ clinically nodepositive status, - missing, $\downarrow$ down-staging according to result of hybrid PET/MRI, $\uparrow$ up-staging according to result of hybrid PET/MRI
MRI, the field of view of the axillary region was not complete [6]. In contrast to Grueneisen, a study by Melsaether et al. showed improved diagnostic accuracy of PET/MR imaging versus PET/CT for axillary nodal staging in breast cancer patients, with an improvement in sensitivity from $88 \%$ to $100 \%$ [21]. Nonetheless, their study differentiated only node-negative from node-

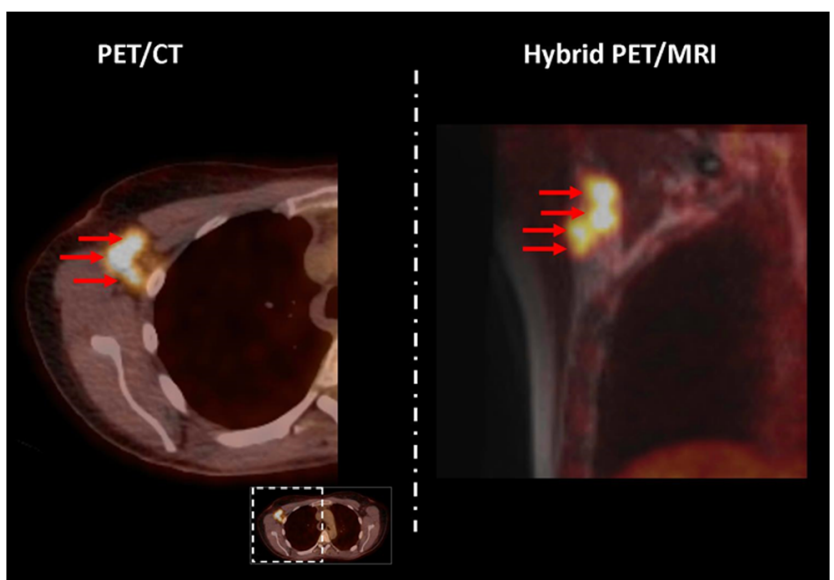

Fig. 1 Example of a patient with a 35-mm invasive carcinoma of no special type in her right breast. One cross-sectional PET/CT image (left) of the right axilla demonstrates three suspicious lymph nodes (red arrows) out of a total of five suspicious lymph nodes on PET/CT. One coronal dedicated axillary hybrid PET/MRI image (right) demonstrates four suspicious lymph nodes (red arrows), with a total of eight suspicious lymph nodes on dedicated axillary hybrid PET/MRI. The most dorsally located suspicious lymph node on (low-dose) PET/CT consists of two suspicious lymph nodes on dedicated axillary hybrid PET/MRI positive disease, rather than considering the number of positive nodes. The results of our study can thus be considered to provide added clinical value compared to standard imaging modalities (US, MRI and PET/CT), further improving a patient-tailored approach for axillary nodal staging in clinically node-positive breast cancer.

The accuracy of MRI for axillary nodal staging in our study may differ from that in standard clinical practice. We used dedicated (coronal) axillary MRI instead of standard (crosssectional) breast MRI that is most commonly used in clinical practice, which might have resulted in higher accuracy than

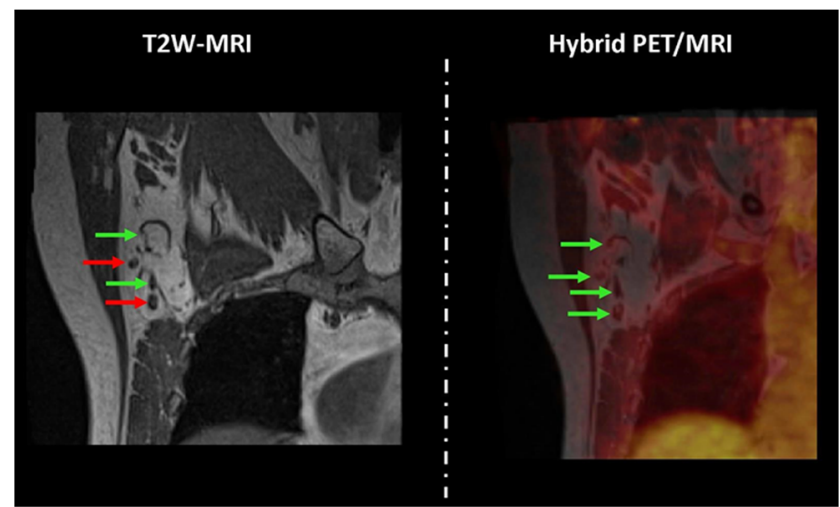

Fig. 2 Example of a patient with a 31-mm invasive carcinoma of no special type in her right breast. A coronal T2-weighted MR image (left) of the right axilla demonstrates two (red arrows) of four suspicious nodes. A coronal dedicated axillary hybrid PET/MRI image (right) demonstrates no FDG-uptake in any of the four nodes (green arrows), which resulted in four negative nodes on dedicated axillary hybrid PET/MRI 
Table 3 Per-patient overview of SUVmax measurements on PET/CT and hybrid PET/MRI

\begin{tabular}{|c|c|c|}
\hline Patient & $\begin{array}{l}\mathrm{PET} / \mathrm{CT} \\
\text { SUVmax }\end{array}$ & $\begin{array}{l}\text { Hybrid PET/MRI } \\
\text { SUVmax }\end{array}$ \\
\hline \multicolumn{3}{|l|}{1} \\
\hline Primary tumor & 9.3 & 12.0 \\
\hline Axillary lymph node & 8.7 & 11.0 \\
\hline \multicolumn{3}{|l|}{2} \\
\hline Primary tumor & 11.1 & 11.7 \\
\hline Axillary lymph node & 16.0 & 20.1 \\
\hline \multicolumn{3}{|l|}{3} \\
\hline Primary tumor & 8.8 & 7.6 \\
\hline Axillary lymph node & 7.7 & 4.6 \\
\hline \multicolumn{3}{|l|}{4} \\
\hline Primary tumor & 5.1 & 10.3 \\
\hline Axillary lymph node & 2.0 & 1.7 \\
\hline \multicolumn{3}{|l|}{5} \\
\hline Primary tumor & 19.5 & 17.1 \\
\hline Axillary lymph node & 11.9 & 8.5 \\
\hline \multicolumn{3}{|l|}{6} \\
\hline Primary tumor & 3.4 & 3.9 \\
\hline Axillary lymph node & 6.6 & 5.7 \\
\hline \multicolumn{3}{|l|}{7} \\
\hline Primary tumor & 1.8 & 1.5 \\
\hline Axillary lymph node & 4.3 & 5.4 \\
\hline \multicolumn{3}{|l|}{8} \\
\hline Primary tumor & 4.0 & 4.5 \\
\hline Axillary lymph node & 3.7 & 5.0 \\
\hline \multicolumn{3}{|l|}{9} \\
\hline Primary tumor & 14.1 & 12.1 \\
\hline Axillary lymph node & 7.9 & 8.0 \\
\hline \multicolumn{3}{|l|}{ Mean } \\
\hline Primary tumor & 8.6 & 9.0 \\
\hline Axillary lymph node & 7.7 & 7.8 \\
\hline
\end{tabular}

Abbreviations: PET positron emission tomography, CT computed tomography, MRI magnetic resonance imaging, SUVmax maximum standard uptake value

with standard breast MRI. A previous systematic review by Kuijs et al. similarly demonstrated superior results for axillary nodal staging when a dedicated axillary MRI protocol was used [7]. However, our study revealed that dedicated axillary hybrid PET/MRI is of added value even compared to dedicated axillary T2W and contrast-enhanced MRI, as it still resulted in changes to clinical nodal status in $75 \%$ and $40 \%$ of cases, respectively. In addition, differences in the performance of $\mathrm{PET} / \mathrm{CT}$ can occur when PET/CT is performed with hanging breast acquisition rather than standard PET/CT. Teixeira et al. demonstrated that PET/CT with hanging breast acquisition improved detection of regional lymph node metastases over standard PET/CT [22].
SUVmax measurements of the primary tumor (8.6 vs. 9.0 ) and the most FDG-avid axillary lymph node (7.7 vs. 7.8 ) in our study were comparable between PET/CT and hybrid PET/ MRI, despite different reconstruction protocols between imaging modalities. Pujara et al. also found a strong correlation in SUVmax between PET/CT and PET/MRI in breast cancer patients, and concluded that SUVs from PET/MRI could be used for quantification of 18F-FDG activity [23]. However, SUVmax comparisons between FDG-avid axillary lymph nodes on PET/CT and PET/MRI were not reported in their study. The results of our study can thus support the earlier findings of Pujara et al. for using SUVmax of FDG-avid axillary nodes on PET/MRI for 18F-FDG activity quantification.

In the future, whole-body PET/MRI, including an integrated dedicated axillary PET/MRI protocol, might replace whole-body PET/CT for locoregional and distant staging in breast cancer, since both locoregional and distant staging seem to be at least as accurate as PET/CT [20, 24-26]. A secondary benefit would be the reduction in radiation exposure to patients of up to $64 \%$ compared to PET/CT [21]. The costeffectiveness of PET/MRI must also be further investigated.

Limitations of the current study include the missing data concerning correlation of dedicated axillary hybrid PET/MR images and final histopathology of axillary lymph nodes; however, nodal metastases were histopathologically confirmed in all patients. Moreover, FDG-avid axillary lymph nodes can be considered malignant, since previous studies have reported high specificity of $95-100 \%$ for the detection of axillary lymph node metastases in breast cancer [16, 27, 28]. However, because of the lack of final histopathology in these cases, it is possible that some benign lymph nodes according to FDG-activity on PET/MR still could have been malignant.

Second, there was a timing difference in the performance of PET/MRI in our cohort. Three of 12 patients underwent only PET/MRI, instead of PET/CT following PET/MRI. This might have led to differences in SUVmax measurements on PET/MRI between the two subgroups [12]. These three patients were thus excluded from subgroup analysis regarding SUVmax measurements on PET/MRI and PET/CT.

Third, patients underwent unenhanced low-dose wholebody PET/CT rather than diagnostic contrast-enhanced PET/ CT. The results of PET/CT should be interpreted with this important limitation in mind.

Finally, hybrid PET/MRI is currently available in only a few hospitals worldwide. Therefore, other techniques should also be further explored to approximate the diagnostic performance of PET/MRI - for instance, by combining hanging breast PET images with MRI images [29].

In conclusion, this feasibility study demonstrated that, compared to standard imaging modalities, dedicated axillary 18F-FDG hybrid PET/MRI appears to improve diagnostic performance for axillary nodal staging in clinically node- 
positive breast cancer patients. Further studies are needed to investigate the accuracy of this hybrid modality and to compare its performance with that of other dedicated imaging tools, such as hanging breast PET/CT.

Funding This study was funded by the Alpe d'Huzes Foundation (Dutch Cancer Society; grant number UM 2013-6229).

\section{Compliance with ethical standards}

Conflict of interest J.E.W. has received institutional research grants from Agfa, Bayer, BRACCO, GE, Philips and Siemens, and speaker honoraria from Bayer and Siemens. F.M.M. has received institution- al grants from Bayer, Siemens and Philips, and speaker honoraria from Bayer. M.L.S. received a speaker honorarium from Roche Nederland B.V. None of these relationships had a direct impact on the current project.

All other authors declare that they have no conflict of interest.

Ethical approval All procedures performed in studies involving human participants were in accordance with the ethical standards of the institutional and/or national research committee and with the 1964 Helsinki declaration and its later amendments or comparable ethical standards.

Informed consent Informed consent was obtained from all individual participants included in the study.

\section{Appendix}

Table 4 Complete overview of all axillary lymph nodes evaluated on (PET)/MRI and $\mathrm{PET} / \mathrm{CT}$ exams

\begin{tabular}{|c|c|c|c|c|c|c|c|c|}
\hline & \multicolumn{2}{|c|}{ T2W MRI } & \multicolumn{2}{|c|}{ CE-T1W MRI } & \multicolumn{2}{|c|}{ Hybrid PET/MRI } & \multicolumn{2}{|l|}{$\mathrm{PET} / \mathrm{CT}$} \\
\hline & $\begin{array}{l}\text { Benign } \\
\text { (score } \\
0-2 \text { ) }\end{array}$ & $\begin{array}{l}\text { Suspicious } \\
\text { (score } \\
3-4)\end{array}$ & $\begin{array}{l}\text { Benign } \\
\text { (score } \\
0-1 \text { ) }\end{array}$ & $\begin{array}{l}\text { Suspicious } \\
\text { (score } \\
2-3 \text { ) }\end{array}$ & $\begin{array}{l}\text { Benign } \\
\text { (score } \\
0-1)\end{array}$ & $\begin{array}{l}\text { Suspicious } \\
\text { (score } \\
2-3 \text { ) }\end{array}$ & $\begin{array}{l}\text { Benign } \\
\text { (score } \\
0-1 \text { ) }\end{array}$ & $\begin{array}{l}\text { Suspicious } \\
\text { (score } \\
2-3 \text { ) }\end{array}$ \\
\hline \multicolumn{9}{|c|}{ Patient } \\
\hline 1 & 2 & 10 & 2 & 10 & 4 & 8 & 1 & 5 \\
\hline 2 & 11 & 0 & 10 & 1 & 9 & 2 & - & - \\
\hline 3 & 15 & 5 & 13 & 7 & 19 & 1 & - & - \\
\hline 4 & 2 & 10 & 3 & 9 & 4 & 8 & 0 & 5 \\
\hline 5 & 4 & 3 & - & - & 0 & 7 & 2 & 9 \\
\hline 6 & 3 & 25 & 17 & 11 & 26 & 2 & 5 & 2 \\
\hline 7 & 6 & 4 & - & - & 7 & 3 & 0 & 4 \\
\hline 8 & 9 & 6 & 8 & 7 & 12 & 3 & 0 & 3 \\
\hline 9 & 4 & 10 & 1 & 13 & 4 & 10 & 0 & 7 \\
\hline 10 & 9 & 2 & 9 & 2 & 10 & 1 & 0 & 1 \\
\hline $11^{*}$ & 5 & 3 & 5 & 5 & 6 & 4 & - & - \\
\hline 12 & 4 & 2 & 3 & 3 & 5 & 1 & 0 & 1 \\
\hline
\end{tabular}

*This concerned the patient with mixed lobular carcinoma with carcinoma of no special type

Abbreviations: $T 2 W$ T2-weighted, MRI magnetic resonance imaging, $C E-T 1 W$ contrast-enhanced T1-weighted, PET positron emission tomography, CT computed tomography
Open Access This article is distributed under the terms of the Creative Commons Attribution 4.0 International License (http:// creativecommons.org/licenses/by/4.0/), which permits unrestricted use, distribution, and reproduction in any medium, provided you give appropriate credit to the original author(s) and the source, provide a link to the Creative Commons license, and indicate if changes were made.

\section{References}

1. Torre LA, Bray F, Siegel RL, Ferlay J, Lortet-Tieulent J, Jemal A. Global cancer statistics, 2012. CA Cancer J Clin. 2015;65:87-108. https://doi.org/10.3322/caac.21262.
2. Howlader N, Noone AM, Krapcho M, Miller D, Bishop K, Altekruse SF, Kosary CL, Yu M, Ruhl J, Tatalovich Z, Mariotto A, Lewis DR, Chen HS, Feuer EJ, Cronin KA (eds). SEER Cancer Statistics Review, 1975-2013, National Cancer Institute. Bethesda, MD, http://seer.cancer.gov/csr/1975_2013/, based on November 2015 SEER data submission, posted to the SEER website, April 2016.

3. Senkus E, Kyriakides S, Ohno S, Penault-Llorca F, Poortmans P, Rutgers E, et al. Primary breast cancer: ESMO clinical practice guidelines for diagnosis, treatment and follow-up. Ann Oncol. 2015;26(Suppl 5):v8-30. https://doi.org/10.1093/annonc/mdv298.

4. Kaufmann M, von Minckwitz G, Mamounas EP, Cameron D, Carey LA, Cristofanilli M, et al. Recommendations from an international consensus conference on the current status and 
future of neoadjuvant systemic therapy in primary breast cancer. Ann Surg Oncol. 2012;19:1508-16. https://doi.org/10. 1245/s10434-011-2108-2.

5. Schipper RJ, van Roozendaal LM, de Vries B, Pijnappel RM, Beets-Tan RG, Lobbes MB, et al. Axillary ultrasound for preoperative nodal staging in breast cancer patients: is it of added value? Breast. 2013;22:1108-13. https://doi.org/10.1016/j. breast.2013.09.002

6. van Nijnatten TJ, Ploumen EH, Schipper RJ, Goorts B, Andriessen $\mathrm{EH}$, Vanwetswinkel S, et al. Routine use of standard breast MRI compared to axillary ultrasound for differentiating between no, limited and advanced axillary nodal disease in newly diagnosed breast cancer patients. Eur J Radiol. 2016;85:2288-94. https://doi.org/10. 1016/j.ejrad.2016.10.030.

7. Kuijs VJ, Moossdorff M, Schipper RJ, Beets-Tan RG, Heuts EM, Keymeulen KB, et al. The role of MRI in axillary lymph node imaging in breast cancer patients: a systematic review. Insights Imaging. 2015;6:203-15. https://doi.org/10.1007/s13244-0150404-2.

8. Koolen BB, Valdes Olmos RA, Elkhuizen PH, Vogel WV, Vrancken Peeters MJ, Rodenhuis S, et al. Locoregional lymph node involvement on 18F-FDG PET/CT in breast cancer patients scheduled for neoadjuvant chemotherapy. Breast Cancer Res Treat. 2012;135:231-40. https://doi.org/10.1007/s10549-012-2179-1.

9. Huellner MW, Appenzeller P, Kuhn FP, Husmann L, Pietsch CM, Burger IA, et al. Whole-body nonenhanced PET/MR versus PET/ $\mathrm{CT}$ in the staging and restaging of cancers: preliminary observations. Radiology. 2014;273:859-69. https://doi.org/10.1148/radiol. 14140090

10. NABON. National breast cancer guideline, Oncoline. 2012. www. oncoline.nl/breastcancer. Accessed 17 May 2017.

11. Neal CH, Daly CP, Nees AV, Helvie MA. Can preoperative axillary US help exclude N2 and N3 metastatic breast cancer? Radiology. 2010;257:335-41. https://doi.org/10.1148/radiol.10100296.

12. Boellaard R, Delgado-Bolton R, Oyen WJ, Giammarile F, Tatsch $\mathrm{K}$, Eschner W, et al. FDG PET/CT: EANM procedure guidelines for tumour imaging: version 2.0. Eur J Nucl Med Mol Imaging. 2015;42:328-54. https://doi.org/10.1007/s00259-014-2961-x.

13. Baltzer PA, Dietzel M, Burmeister HP, Zoubi R, Gajda M, Camara $\mathrm{O}$, et al. Application of MR mammography beyond local staging: is there a potential to accurately assess axillary lymph nodes? Evaluation of an extended protocol in an initial prospective study. AJR Am J Roentgenol. 2011;196:W641-7. https://doi.org/10.2214/ AJR.10.4889.

14. Farshchian N, Tamari S, Farshchian N, Madani H, Rezaie M, Mohammadi-Motlagh HR. Diagnostic value of chemical shift artifact in distinguishing benign lymphadenopathy. Eur J Radiol. 2011;80:594-7. https://doi.org/10.1016/j.ejrad.2010.10.005.

15. Lambregts DM, Heijnen LA, Maas M, Rutten IJ, Martens MH, Backes WH, et al. Gadofosveset-enhanced MRI for the assessment of rectal cancer lymph nodes: predictive criteria. Abdom Imaging. 2013;38:720-7. https://doi.org/10.1007/s00261-012-9957-4.

16. Aukema TS, Straver ME, Peeters MJ, Russell NS, Gilhuijs KG, Vogel WV, et al. Detection of extra-axillary lymph node involvement with FDG PET/CT in patients with stage II-III breast cancer. Eur J Cancer. 2010;46:3205-10. https://doi.org/10.1016/j.ejca. 2010.07.034

17. Gradishar WJ, Anderson BO, Balassanian R, Blair SL, Burstein HJ, Cyr A, et al. Invasive breast cancer version 1.2016, NCCN clinical practice guidelines in oncology. J Natl Compr Cancer Netw. 2016;14:324-54

18. Giuliano AE, Ballman K, McCall L, Beitsch P, Whitworth PW, Blumencranz $\mathrm{P}$, et al. Locoregional recurrence after sentinel lymph node dissection with or without Axillary dissection in patients with sentinel lymph node metastases: long-term follow-up from the American College of Surgeons Oncology Group (Alliance) ACOSOG Z0011 randomized trial. Ann Surg. 2016;264:413-20. https://doi.org/10.1097/SLA.0000000000001863.

19. Teixeira SC, Koolen BB, Elkhuizen PH, Vrancken Peeters MT, Stokkel MP, Rodenhuis S, et al. PET/CT with 18F-FDG predicts short-term outcome in stage II/III breast cancer patients upstaged to N2/3 nodal disease. Eur J Surg Oncol. 2017;43:625-35. https://doi. org/10.1016/j.ejso.2016.10.012.

20. Grueneisen J, Nagarajah J, Buchbender C, Hoffmann O, Schaarschmidt BM, Poeppel T, et al. Positron emission tomography/magnetic resonance imaging for local tumor staging in patients with primary breast cancer: a comparison with positron emission tomography/computed tomography and magnetic resonance imaging. Investig Radiol. 2015;50:505-13. https://doi.org/ 10.1097/RLI.0000000000000197.

21. Melsaether AN, Raad RA, Pujara AC, Ponzo FD, Pysarenko KM, Jhaveri K, et al. Comparison of whole-body (18)F FDG PET/MR imaging and whole-body (18)F FDG PET/CT in terms of lesion detection and radiation dose in patients with breast cancer. Radiology. 2016;281:193-202. https://doi.org/10.1148/radiol. 2016151155.

22. Teixeira SC, Koolen BB, Vogel WV, Wesseling J, Stokkel MP, Vrancken Peeters MJ, et al. Additional prone 18F-FDG PET/CT acquisition to improve the visualization of the primary tumor and regional lymph node metastases in stage II/III breast cancer. Clin Nucl Med. 2016;41:e181-6. https://doi.org/10.1097/RLU. 0000000000001101.

23. Pujara AC, Raad RA, Ponzo F, Wassong C, Babb JS, Moy L, et al. Standardized uptake values from PET/MRI in metastatic breast cancer: an organ-based comparison with PET/CT. Breast J. 2016;22:264-73. https://doi.org/10.1111/tbj.12569.

24. Pace L, Nicolai E, Luongo A, Aiello M, Catalano OA, Soricelli A, et al. Comparison of whole-body PET/CT and PET/MRI in breast cancer patients: lesion detection and quantitation of 18F-deoxyglucose uptake in lesions and in normal organ tissues. Eur J Radiol. 2014;83:289-96. https://doi.org/ 10.1016/j.ejrad.2013.11.002.

25. Sawicki LM, Grueneisen J, Schaarschmidt BM, Buchbender C, Nagarajah J, Umutlu L, et al. Evaluation of (18)F-FDG PET/ MRI, (18)F-FDG PET/CT, MRI, and CT in whole-body staging of recurrent breast cancer. Eur J Radiol. 2016;85:459-65. https:// doi.org/10.1016/j.ejrad.2015.12.010.

26. Segaert I, Mottaghy F, Ceyssens S, De Wever W, Stroobants S, Van Ongeval C, et al. Additional value of PET-CT in staging of clinical stage IIB and III breast cancer. Breast J. 2010;16:617-24. https:// doi.org/10.1111/j.1524-4741.2010.00987.x.

27. Fuster D, Duch J, Paredes P, Velasco M, Munoz M, Santamaria G, et al. Preoperative staging of large primary breast cancer with [18F]fluorodeoxyglucose positron emission tomography/ computed tomography compared with conventional imaging procedures. J Clin Oncol. 2008;26:4746-51. https://doi.org/10.1200/ JCO.2008.17.1496.

28. Veronesi U, De Cicco C, Galimberti VE, Fernandez JR, Rotmensz $\mathrm{N}$, Viale G, et al. A comparative study on the value of FDG-PET and sentinel node biopsy to identify occult axillary metastases. Ann Oncol. 2007;18:473-8. https://doi.org/10.1093/annonc/mdl425.

29. Teixeira SC, Rebolleda JF, Koolen BB, Wesseling J, Jurado RS, Stokkel MP, et al. Evaluation of a hanging-breast PET system for primary tumor visualization in patients with stage I-III breast cancer: comparison with standard PET/CT. AJR Am J Roentgenol. 2016;206:1307-14. https://doi.org/10.2214/AJR.15.15371. 